\title{
ON A NAVIER-STOKES TYPE EQUATION AND INEQUALITY
}

\author{
GIOVANNI PROUSE \\ Dipartimento di Matematica del Politecnico di Milano \\ Piazza Leonardo da Vinci, 32, 20133 Milano, Italy
}

\begin{abstract}
A Navier-Stokes type equation corresponding to a non-linear relationship between the stress tensor and the velocity deformation tensor is studied and existence and uniqueness theorems for the solution, in the 3-dimensional case, of the Cauchy-Dirichlet problem, for a bounded solution and for an almost periodic solution are given. An inequality which in some sense is the limit of the equation is also considered and existence theorems for the solution of the Cauchy-Dirichlet problems and for a periodic solution are stated.
\end{abstract}

1. Introduction. I wish to present some results regarding a Navier-Stokes type equation and its corresponding inequality. The results will only be stated here; for their proofs, the reader is referred to the original papers.

The starting point for the introduction of the Navier-Stokes type equation I shall consider, which is a modification of the classical Navier-Stokes equation for an incompressible fluid, is the observation that, for large values of the velocity, there is no experimental evidence that the linear relationship between the stress tensor and the deformation velocity tensor (on which the classical Navier-Stokes equations are based) continues to hold. It does not therefore seem unreasonable to substitute this relationship with one which is not linear when the velocity is "large".

The choice of this relationship is made in such a way that (i) it is physically significant, (ii) it reduces to the classical one when the velocity is "not large", (iii) for the corresponding Navier-Stokes type equation a global existence and uniqueness theorem holds for the solution, in the 3-dimensional case, of the Cauchy-Dirichlet problem $\left({ }^{1}\right)$.

Let $\sigma(\xi)$ be a function, defined for $0 \leq \xi \leq \bar{\xi}, \bar{\xi} \leq+\infty$, satisfying the following conditions:

$\left({ }^{1}\right)$ For the Navier-Stokes equations, this theorem is known only for plane flows. 
(a) $\sigma(\xi) \in C^{1}[0, \infty), \sigma(\xi) \geq \mu>0, \sigma^{\prime}(\xi) \geq 0$;

$\left(\mathrm{b}_{1}\right)$ if $\bar{\xi}=+\infty$ and $M>0$ is an arbitrary fixed value, then $\sigma(\xi) \geq \alpha_{1} \xi^{3}$ when $\xi \geq M\left(\alpha_{1}>0\right)$

$\left(\mathrm{b}_{2}\right)$ if $\bar{\xi}<+\infty$, then $\lim _{\xi \rightarrow \bar{\xi}^{-}} \sigma(\xi)=+\infty$.

I shall assume that the relationship between the stress tensor $T=\left\{\tau_{i j}\right\}$ and the deformation velocity tensor $S=\left\{\eta_{i j}\right\}=\left\{\frac{1}{2}\left(\partial u_{i} / \partial x_{j}+\partial u_{j} / \partial x_{i}\right)\right\}\left(u_{i}\right.$ being the components of the velocity) is given by

$$
\tau_{i j}=-p \delta_{i j}+\frac{1}{2}\left(\frac{\partial \phi_{i}(\mathbf{u})}{\partial x_{j}}+\frac{\partial \phi_{j}(\mathbf{u})}{\partial x_{i}}\right)
$$

having denoted by $p$ the pressure and setting

$$
\phi_{i}(\mathbf{u})=\sigma(|\mathbf{u}|) u_{i} .
$$

It is obvious that, if $\sigma(\xi)=\mu$ (viscosity of the fluid), (1.1) reduces to the classical linear law. Introducing now (1.1) into the general equations of conservation of momentum and assuming the fluid is incompressible, the following Navier-Stokes type equations are obtained:

$$
\left\{\begin{array}{l}
\partial \mathbf{u} / \partial t-\Delta(\sigma(|\mathbf{u}|) \mathbf{u})+(\mathbf{u} \cdot \nabla) \mathbf{u}-\nabla p+\nabla(\operatorname{div}(\sigma(|\mathbf{u}|) \mathbf{u})=\mathbf{f}, \\
\operatorname{div} \mathbf{u}=0
\end{array}\right.
$$

to which I shall always refer in Sections $2-4$, devoted to the study of various problems connected with these equations, according to the following plan.

In Section 2 I shall introduce some basic notations and give the definition of weak solution; a lemma, essentially due to Prodi [6], will also be stated, since it plays an essential role in the proof of most of the results obtained.

Section 3 is devoted to the statements of existence and uniqueness theorems for the global solution of the Cauchy-Dirichlet problem in the 3-dimensional case and of existence and uniqueness theorems for a solution bounded on $J=(-\infty,+\infty)$; the latter results are due to Iannelli [5], the former to Prouse [7].

In Section 4 the almost-periodicity of the bounded solution is investigated and the existence and uniqueness of an almost-periodic solution is proved under the assumption that $\mathbf{f}(t)$ is almost-periodic and "sufficiently small" (depending on $\Omega$, $\left.\mu, \alpha_{1}\right)$. This theorem is due to Iannelli [5].

Finally, in Section 5, a Navier-Stokes type inequality associated with (1.3) is studied. It is shown, first, that this inequality can be considered as a "limit" case of equations (1.3) when the function $\sigma(\xi)$ tends to the "limit" function

$$
\widehat{\sigma}(\xi)= \begin{cases}\sigma(\xi) & \text { when } 0 \leq \xi \leq M \\ +\infty & \text { when } \xi>M\end{cases}
$$

and a global existence and uniqueness theorem is proved for the solution of the Cauchy-Dirichlet problem. Subsequently, the periodicity of the solutions is investigated and the existence and uniqueness of a periodic solution with the same period as the known term is proved.

The results of this section are due to Collini [2], [3]. 
2. Basic notations and definitions. Let $\Omega$ be an open, bounded set with boundary of class $C^{2}{ }^{2}$ ) and let $\mathcal{N}$ be the set of $C^{\infty}$ vectors with compact support and null divergence in $\Omega$. I shall denote by $N^{0}$ and $N^{1}$ respectively the closures of $\mathcal{N}$ in $L^{2}(\Omega)$ and in $H^{1}(\Omega)$ and by $N^{-1}$ the dual of $N^{1}$.

The scalar products in $N^{0}, N^{1}, N^{-1}$ are defined respectively by

$$
\begin{aligned}
(\mathbf{u}, \mathbf{v})_{N^{0}} & =(\mathbf{u}, \mathbf{v})_{L^{2}(\Omega)}, \\
(\mathbf{u}, \mathbf{v})_{N^{1}} & =(\mathbf{u}, \mathbf{v})_{H_{0}^{1}(\Omega)}=\left((-\Delta)^{1 / 2} \mathbf{u},(-\Delta)^{1 / 2} \mathbf{v}\right)_{L^{2}(\Omega)}, \\
(\mathbf{u}, \mathbf{v})_{N^{-1}} & =(\mathbf{u}, \mathbf{v})_{H^{-1}(\Omega)}=\left(G^{1 / 2} \mathbf{u}, G^{1 / 2} \mathbf{v}\right)_{L^{2}(\Omega)}
\end{aligned}
$$

where $G$ is Green's operator, from $N^{-1}$ to $N^{1}$ relative to $-\Delta$.

Assuming that $\mathbf{f}(t) \in L^{2}\left(0, T ; L^{1}\right)$, the vector $\mathbf{u}$ will be called a weak solution of (1.3) in $Q=\Omega \times(0, T)$ satisfying a homogeneous Dirichlet boundary condition if

(i) $\mathbf{u}(t) \in L^{2}\left(0, T ; N^{1}\right) \cap L^{5}\left(0, T ; L^{5}\right) \cap H^{1}\left(0, T ; L^{2}\right)$ and $\Delta(\sigma(|\mathbf{u}|) \mathbf{u}(t)) \in$ $L^{2}\left(0, T ; L^{2}\right)$;

(ii) $\mathbf{u}$ satisfies, $\forall \mathbf{h}(t) \in L^{2}\left(0, T ; H^{2} \cap N^{1}\right)$, the equation

$$
\int_{0}^{T} \int_{\Omega}\left(\left(\frac{\partial \mathbf{u}}{\partial t}+(\mathbf{u} \cdot \nabla) \mathbf{u}-\mathbf{f}\right) \cdot \mathbf{h}-\phi(\mathbf{u}) \cdot \Delta \mathbf{h}\right) d \Omega d t=0 .
$$

If we assume that $\sigma(\xi)$ satisfies, in addition to (a), $\left(\mathrm{b}_{1}\right)$, the condition

(c) $\alpha_{1} \xi^{s-1} \leq \sigma(\xi) \leq \alpha_{2} \xi^{s-1}\left(\xi \geq M, \alpha_{1}, \alpha_{2}>0, s \geq 4\right)$,

i.e. if the asymptotic behaviour of $\sigma(\xi)$ is of polynomial type, of degree $\geq 3$, then the definition of solution given above can be substituted by the weaker one:

$\left(\mathrm{i}^{\prime}\right) \mathbf{u}(t) \in L^{\infty}\left(0, T ; N^{0}\right) \cap L^{s+1}\left(0, T ; L^{s+1}\right) \cap H^{1,(s+1) / s}\left(0, T ;\left(H^{2, s+1}\right)^{\prime}\right) \cap$ $L^{2}\left(0, T ; N^{1}\right)$;

(ii') u satisfies, $\forall \mathbf{h}(t) \in L^{\infty}\left(0, T ; N^{0}\right) \cap L^{s+1}\left(0, T ; H^{2, s+1}\right) \cap L^{2}\left(0, T ; N^{1}\right)$, the equation

$$
\left\langle\mathbf{u}^{\prime}-\Delta \phi(\mathbf{u})+(\mathbf{u} \cdot \nabla) \mathbf{u}-\mathbf{f}, \mathbf{h}\right\rangle=0 .
$$

The corresponding definitions of solution on $J=(-\infty,+\infty)$ follow directly from the preceding ones, on substituting the interval $(0, T)$ with a generic interval $(a, b)$ of the $t$-axis.

As already mentioned, most of the proofs of the theorems stated in the next three sections are based on the following lemma, due essentially to Prodi.

Lemma. Let $\mathbf{u} \in N^{0}, \mathbf{v} \in L^{5}(\Omega)$; then, $\forall \mu>0$,

$$
|\langle(\mathbf{u} \cdot \nabla) \mathbf{v}, G \mathbf{u}\rangle| \leq \frac{\mu}{4}\|\mathbf{u}\|_{L^{2}}^{2}+C\|\mathbf{v}\|_{L^{5}}^{5}\|\mathbf{u}\|_{N^{-1}}^{2},
$$

where $C$ depends only on $\mu$ and $\Omega$.

$\left({ }^{2}\right)$ This assumption is made for the sake of simplicity; the results hold under much more general conditions on $\Omega$ (see [4]). 
3. Existence and uniqueness theorems. Consider, first, the Cauchy problem, assigning the value of $\mathbf{u}$ at the time $t=0$ :

$$
\mathbf{u}(0)=\mathbf{u}_{0} .
$$

The following existence and uniqueness theorems hold:

THEOREM 3.1. Assume that $\mathbf{f}(t) \in H^{1}\left(0, T ; N^{-1}\right)$, that $\mathbf{u}_{0} \in N^{1} \cap L^{\infty}$ and that $\sigma(\xi)$ satisfies $(\mathrm{a}),\left(\mathrm{b}_{1}\right)$ or $(\mathrm{a}),\left(\mathrm{b}_{2}\right)$. There exists then a unique vector $\mathbf{u}$ satisfying (i), (ii). If ( $\left.\mathrm{b}_{2}\right)$ holds then $|\mathbf{u}|<\bar{\xi}$.

Theorem 3.2. Assume that $\mathbf{f}(t) \in L^{1}\left(0, T ; N^{-1}\right)$, that $\mathbf{u}_{0} \in N^{0}$ and that $\sigma(\xi)$ satisfies (a), (c). There exists then a unique vector $\mathbf{u}$ satisfying $\left(\mathrm{i}^{\prime}\right)$, (ii').

Regarding the problem of bounded solutions, we have

Theorem 3.3. Assume that

$$
\sup _{t \in J} \int_{t}^{t+1}\|\mathbf{f}(\eta)\|_{L^{2}} d \eta=K_{1}<+\infty, \quad \sup _{t \in J} \int_{t}^{t+1}\left\|\mathbf{f}^{\prime}(\eta)\right\|_{N^{-1}} d \eta=K_{2}<+\infty .
$$

Then, if $K_{1}$ is sufficiently small (depending only on $\Omega, \mu, \alpha_{1}$ ) there exists a unique solution $\mathbf{u}(t)$ which is bounded on $J$, i.e. such that

$$
\sup _{t \in J}\|\mathbf{u}\|_{L^{2}\left(t, t+1 ; N^{1}\right) \cap L^{5}\left(t, t+1 ; L^{5}\right) \cap H^{1}\left(t, t+1 ; N^{0}\right)} \leq M_{1}<+\infty .
$$

4. Almost-periodic solutions. Almost-periodicity will always be understood in the sense of Bohr, i.e. a function $v(t) \in C^{0}(J ; B)$ (where $B$ is a Banach space) will be called $B$-almost-periodic if, $\forall \varepsilon>0$, there exists a relatively dense set of numbers $\{\tau\}_{\varepsilon}$ such that, $\forall \tau \in\{\tau\}_{\varepsilon}$,

$$
\sup _{t \in J}\|v(t+\tau)-v(t)\|_{B}<\varepsilon \text {. }
$$

The elements of the set $\{\tau\}_{\varepsilon}$ are called the $\varepsilon$-almost-periods of $v(t)$.

If $\langle v(t), h\rangle$ is almost-periodic $\forall h \in B^{\prime}$, then $v(t)$ is said to be $B$-weakly almostperiodic.

The results regarding almost-periodicity are closely related to the existence and uniqueness of a bounded solution $\left(^{3}\right)$; from Theorem 3.3 follows, in fact, an almost-periodicity result, given by

THEOREM 4.1. Assume that $\mathbf{f}(t), \mathbf{f}^{\prime}(t)$ are respectively $L^{2}\left(0,1 ; N^{0}\right)$ - and $L^{2}\left(0,1 ; N^{-1}\right)$-weakly almost-periodic, i.e. that, for all $\varepsilon>0$ and $h_{1} \in L^{2}(t, t+$ $\left.1 ; N^{0}\right), h_{2} \in L^{2}\left(t, t+1 ; N^{-1}\right)$, there exist two relatively dense sets $\left\{\tau_{h_{1}}\right\}_{\varepsilon},\left\{\tau_{h_{2}}\right\}_{\varepsilon}$ such that

$$
\sup _{t \in J} \int_{t}^{t+1}\left(\mathbf{f}\left(\eta+\tau_{h_{1}}\right)-\mathbf{f}(\eta), \mathbf{h}_{1}(\eta)\right)_{N^{0}} d \eta<\varepsilon \quad \forall \tau_{h_{1}} \in\left\{\tau_{h_{1}}\right\}_{\varepsilon},
$$

$\left({ }^{3}\right)$ For general results on almost-periodic functional equations see, for instance, [1]. 


$$
\sup _{t \in J} \int_{t}^{t+1}\left(\mathbf{f}^{\prime}\left(\eta+\tau_{h_{2}}\right)-\mathbf{f}^{\prime}(\eta), \mathbf{h}_{2}(\eta)\right)_{N^{-1}} d \eta<\varepsilon \quad \forall \tau_{h_{2}} \in\left\{\tau_{h_{2}}\right\}_{\varepsilon} .
$$

Assume, moreover, that the first equality of (3.2) holds with $K_{1}$ sufficiently small. There exists then a unique solution $\mathbf{u}(t)$ which is $L^{2}\left(0,1 ; N^{0}\right)$-almost-periodic.

5. A Navier-Stokes type inequality. Assume that the relationship between the stress tensor and the deformation velocity tensor is formally defined by (1.1), with $\phi(\mathbf{u})$ given by (1.2), (1.4). This is obviously a "limit" case to the ones considered in the preceding sections and physically corresponds to the assumption of the existence of a "barrier" which does not allow the velocity of the fluid to exceed the value $M$. Since equations (1.3) lose their meaning in this case, it is natural to try to define the solution of this problem as the limit, as $n \rightarrow \infty$, of a sequence $\left\{\mathbf{u}_{n}\right\}$ of solutions of equations (1.3) corresponding to functions $\sigma_{n}(\xi)$ which approximate $\widehat{\sigma}(\xi)$ given by $(1.4)$.

It can be shown that this is actually possible, since the whole sequence $\left\{\mathbf{u}_{n}\right\}$ converges, in an appropriate topology, to the unique solution of the inequality $\left({ }^{4}\right)$

$$
\left\langle\mathbf{u}^{\prime}-\Delta(\widehat{\sigma}(|\mathbf{u}|) \mathbf{u})+(\mathbf{u} \cdot \nabla) \mathbf{u}-\mathbf{f}, G(\mathbf{u}-\mathbf{h})\right\rangle \leq 0
$$

satisfying the initial condition (3.1). The solution obtained in this way is, moreover, independent of the approximating sequence $\left\{\mathbf{u}_{n}\right\}$. Inequality (5.1) can therefore be interpreted as the limit of equations (1.3).

Assuming that $\mathbf{f}(t) \in L_{\text {loc }}^{2}\left(J ; L^{2}\right)$ and is periodic with period $T$, it can be proved that there exists a unique solution of (5.1) which is periodic with the same period $T$.

\section{References}

[1] L. Amerio and G. Prouse, Almost-periodic Functions and Functional Equations, Van Nostrand, 1971.

[2] T. Collini, On a Navier-Stokes type inequality, Rend. Ist. Lomb. Sc. Lett., to appear.

[3] -, Periodic solutions of a Navier-Stokes type inequality, ibid., to appear.

[4] P. Grisvard, Elliptic Problems in Nonsmooth Domains, Pitman, 1985.

[5] A. Iannelli, Bounded and almost-periodic solutions of a Navier-Stokes type equation, Rend. Accad. Naz. Sci. XL, to appear.

[6] G. Prodi, Un teorema di unicità per le equazioni di Navier-Stokes, Ann. Mat. Pura Appl. 48 (1959), 173-182.

[7] G. Prouse, On a Navier-Stokes type equation, in: Non-linear Analysis: a Tribute to G. Prodi, Quaderni Scuola Norm. Sup. Pisa, to appear.

$\left({ }^{4}\right)$ The definitions of solution of the inequality are analogous to (i), (ii), or (i'), (ii'), with the exception that now both $\mathbf{u}$ and $\mathbf{h}$ must also belong, a.e. on $(0, T)$, to the closed convex set $K=\left\{\mathbf{v} \in L^{\infty}(\Omega) ;|\mathbf{v}| \leq M\right\}$. 